\title{
The anterior limb of the anterior commissure is an access route to contralaterally stored olfactory preference memories
}

\author{
DAVID KUCHARSKI, NANCY BURKA, and W. G. HALL \\ Duke University, Durham, North Carolina
}

\begin{abstract}
Young rats learn to prefer novel odors that have been paired with reinforcers. Memories for such preference learning can be confined to one side of the olfactory system by blocking the contralateral naris during odor-reinforcer training. These memories can be accessed only from the trained naris in 6-day-old pups. But in pups 12 days of age and older, these memories, which are still stored only on one side, can be accessed from either the trained or the untrained naris. The ability of 12-day-olds to access unilaterally stored memories from the untrained side of the olfactory system permitted us to further explore the locations of olfactory memories and the routes of access to these memories. We report here that when preference memories were tested from the untrained side after selective fiber-tract transections on the trained side: (1) transection of the anterior limb of the anterior commissure at various rostral-caudal levels prevented retrieval of odor memories; (2) transection of the posterior limb did not disrupt memory access; and (3) transection of the olfactory peduncle on the trained side, thereby removing the contribution of the trained olfactory bulb, did not eliminate access to odor-preference memories. These findings suggest that access to contralateral olfactory memories is subserved by the anterior limb of the anterior commissure, probably through access to its most distal projections to the anterior olfactory nucleus and the anterior piriform cortex, and that retrieval of preference memories does not require access to the trained olfactory bulb.
\end{abstract}

Rat pups will learn to prefer a novel, normally aversive odor as a result of pairing that odor with a reinforcer such as oral infusions of milk or sucrose (Johanson \& Hall, 1982; Johanson \& Teicher, 1980) or other arousing stimulation (Pedersen, Williams, \& Blass, 1982; Sullivan \& Hall, 1988; Sullivan, Hofer, \& Brake, 1986; Sullivan \& Leon, 1987). In pups 6 days of age and younger, storage of and access to such preference memories can be confined to one side of the olfactory system by occluding the contralateral naris at the time of initial training (Kucharski, Johanson, \& Hall, 1986). Six-day-old pups trained in this fashion subsequently showed a preference for the odor when tested with the same naris open that was open during training (trained naris) and showed a normal aversion to the odor when tested with the contralateral (untrained) naris open. However, when the same experiment was carried out in 12-day-old pups, the odorpreference memory was retrieved (i.e., preference was shown) from both the trained and the untrained naris (Kucharski \& Hall, 1987, 1988).

This research was supported by USPHS Fellowship MH09436 to D.K., and by NICHD Grant HD17458 and NIMH Research Scientist Development Award MH00571 to W.G.H. We are grateful to C. Anderson, L. F. Cierpial, and S. E. Swithers for their helpful comments on earlier versions of this paper. David Kucharski died in an automobile accident on October 24, 1987, shortly after completion of this work. Correspondence may be addressed to W. G. Hall, Department of Psychology, Duke University, Durham, NC 27706.
The major olfactory cross projections potentially mediating this transfer of learning are carried in the anterior commissure (AC; e.g., see Haberly \& Price, 1978; Luskin \& Price, 1983a, 1983b). Between 6 and 12 days of age, several of these cross projections reach their contralateral targets (Schwob \& Price, 1984). This maturation of fiber systems of the AC appears to be responsible for the bilateral memory access in 12-day-olds, since transection of the $\mathrm{AC}$ before training prevented the expression of an odor preference when the untrained naris was tested (Kucharski \& Hall, 1987, 1988).

Despite the behavioral expression of bilateral preference at 12 days of age, preference memories were still stored unilaterally after unilateral training. This unilateral storage of preference memories in older pups was revealed by the finding that $\mathrm{AC}$ transections made $1 \mathrm{~h}$ after training eliminated the learned preference shown from the untrained side (Kucharski \& Hall, 1987, 1988). If memories had been initially encoded bilaterally, AC transections after training would be expected to have left learned preference intact on both sides.

This finding of bilaterally accessible but unilaterally stored memories creates an experimental opportunity for exploring neural systems for olfactory memory. Odor stimulation of the untrained side of the olfactory system can be used as a probe of memory systems on the trained side. Because the memory is not present on the untrained side in unilaterally trained pups, neural pathways to the trained-side memories can be disrupted and their impor- 
tance in memory recall can be evaluated. A similar strategy has been used in the analysis of memory substrates in primates using split-brain preparations (e.g., Doty, 1973).

We have also taken advantage of a naturally maturing system in which developmental change in behavior and anatomy provides opportunities to explore potential routes of access to preference memories. These opportunities arise from the correlation between the behaviorally demonstrated change in access to contralateral memories between 6 and 12 days of age and Schwob and Prices's (1984) description of the maturation of several contralateral olfactory projections crossing in the AC. Using ${ }^{3} \mathrm{H}$-leucine tracing techniques, those investigators found that fibers of three systems appear to reach their targets in Layer $\mathrm{Ib}$ of the contralateral olfactory cortex sometime after 6 but before 12 days of age. Although these findings do not confirm the actual establishment of synapses, they do provide an indication of the first potential functional contact of specific projections. These projections include (1) fibers from the anterior olfactory nucleus (AON) to the AON on the contralateral side, (2) fibers from the AON to the contralateral anterior piriform cortex ( $\mathrm{PCa})$, and (3) fibers from the $\mathrm{PCa}$ to the contralateral posterior piriform cortex (PCp). Fibers of the first two projections are carried in the anterior limb of the $\mathrm{AC}$ (Figure 1), whereas fibers in the third projection reach their targets via the posterior limb of the $\mathrm{AC}$.

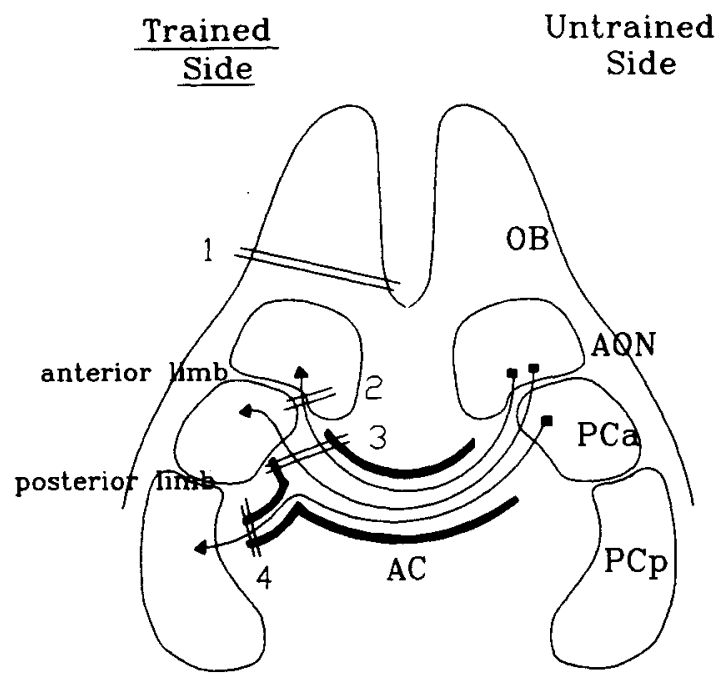

Figure 1. Schematic of anterior commissure (AC) projections from the untrained (right) to the trained (left) side of the olfactory system showing locations of transections in this study. These projections are those that mature between 6 and 12 days of age (Schwob \& Price, 1984), and are thus the candidate routes for the new access to olfactory memories that develops in 12-day-old pups (Kucharski \& Hall, 1987). Four levels of transection are also indicated: (1) transection of the olfactory peduncle, ( 2 and 3 ) transections of the ascending limb of the $\mathrm{AC}$, and (4) transection of the posterior limb of the AC. AON = anterior olfactory nucleus, $\mathrm{PCa}=$ anterior piriform cortex, $\mathrm{PCp}=$ posterior piriform cortex, $\mathrm{OB}=$ olfactory bulb.
In the present study, we used knife-cut transections of the anterior and posterior limbs of the AC to determine which of these pathways are critical for contralateral access to preference memories and to further assess whether the presence of olfactory structures distal to the AON is necessary for the expression of a learned odor preference. The logic for these transections is detailed in Figure 1. Transections of the olfactory peduncle at Location 1 eliminate projections to the trained olfactory bulb and, to the extent that they are complete, outputs from it. Cuts within the region indicated by Locations 2 and 3 (varied levels of the anterior limb of the $\mathrm{AC}$ ) disrupt the projections to the AON and the PCa. Cuts at Location 4 (posterior limb of the AC) disrupt the projection to the PCp. In brief, we found that only transections in region 2-3 (ascending limb of the AC) disrupted access to memories from the untrained side.

\section{Subjects}

\section{METHOD}

Pups were 12-day-old (male and female) progeny of Charles River CD-strain rats mated in our laboratory. Pregnant females were housed in individual cages $(45 \times 24 \times 20 \mathrm{~cm})$ containing woodchip bedding (Ab-Sorb-Dri, Mayfield, NJ). Water and Formulab pellets (Purina; St. Louis, MO) were continuously available. The colony room was maintained at $21^{\circ}-24^{\circ} \mathrm{C}(40 \%-80 \%$ relative humidity) and was on a 14:10 h light:dark cycle. Females were checked daily for births at $1700 \mathrm{~h}$, and pups found at that time were termed 0 days of age. At 2 days of age, litters were culled to 10 pups each, with an equal number of males and females.

\section{Deprivation Procedures}

Pups were removed from their mothers' cages $18 \mathrm{~h}$ before training and were thus deprived of maternal care as well as the opportunity to suckle and receive milk. During deprivation, they were housed individually in Styrofoam containers $(10 \mathrm{~cm}$ diam.) inside a warm $\left(32.5^{\circ} \pm 0.5^{\circ} \mathrm{C}\right)$, moist $(70 \%-90 \%$ relative humidity $)$ incubator (Isolette, Air-Shields; Hatboro, PA).

\section{Oral Cannulas}

Oral cannulas used to deliver sucrose rewards (see Hall, 1979, for details) were installed at the time of deprivation. The cannulas were constructed by heating one end of a $10-\mathrm{cm}$ piece of fine polyethylene tubing (PE-10; Clay-Adams) and, as the end began to blister, pressing it flat on a smooth surface, forming a small flange. The pups were lightly anesthetized with methoxyflurane (Metofane, Pittman Moore; Washington Crossing, NJ); the cannula was then implanted by first inserting a thin stainless steel wire between the angle of the mandibles and up through the soft tissue of the digastric muscles and tongue. The tip of the wire was maneuvered out of the mouth, where the nonflanged end of the cannula was frictionfitted over it. As the wire was pulled back out from the lower jaw, the cannula was pulled into position on the back of the tongue (where the infusion of sucrose would be delivered). The implantation procedure required less than $10 \mathrm{sec}$, and the pups appeared to habituate rapidly to the presence of the cannula.

\section{Nose Plug for Reversible Unilateral Olfactory Occlusion}

To restrict olfactory stimulation to one nasal cavity, a soft rubber plug was inserted into the opposite naris. The plug was made from an 8.0-mm length of Silastic tubing (Dow Corning; $0.94 \mathrm{~mm}$ outside diam., beveled at the insertion end) with a knotted thread (No. 0 surgical silk) placed in the lumen of the tube to block airflow and to serve as an aid in removal. The plug, with its tip lubricated with petroleum jelly, was gently inserted with forceps while 
the pup was held in the experimenter's hand. The pups appeared to adapt to the presence of the plug quickly and paid it little attention. For all procedures, $30 \mathrm{~min}$ was allowed after plug insertion before training or testing.

\section{Transections}

One hour after training, the pups were anesthetized with methoxyflurane (Metofane). The skull was exposed on the midline and a burr hole was made at a site appropriate for the transection. The transection was made with a 30-ga hypodermic needle, rounded at the tip and inserted through the burr hole to a predetermined depth. Transections were made as follows (see Figure 1): (1) for olfactory peduncle cuts, the needle was inserted just lateral to the sagittal sinus, $3.5 \mathrm{~mm}$ anterior to bregma, and angled $15^{\circ}$ forward from perpendicular; it was carefully passed to the base of the cranium, swung across the bulb in the frontal plane, and drawn up the lateral wall as it was withdrawn; ( 2 and 3 ) for anterior limb lesions, the needle was inserted $1 \mathrm{~mm}$ lateral and 1-2 mm anterior to bregma, to a depth of $5.5 \mathrm{~mm}$, and swung through a $30^{\circ}$ arc in a frontal plane perpendicular to the skull; (4) for posterior limb transections, the needle was inserted $2 \mathrm{~mm}$ lateral and $2 \mathrm{~mm}$ posterior to bregma, to a depth of $7 \mathrm{~mm}$, and swung through a $30^{\circ}$ arc in a plane oriented $30^{\circ}$ from sagittal (also see histology). The skin incisions were closed with Vetbond (Animal Care Products, 3M; St. Paul, MN). For sham transections, the needle was inserted only into the cortex at a site corresponding to the particular transection. The side of transection was counterbalanced in accord with side of training (all transections were made on the trained side). The pups recovered rapidly from this surgery and were tested for odor preference $4 \mathrm{~h}$ later.

We chose to use transections of the peduncle to create a functional bulbectomy in preference to an aspiration lesion (which might have been preferable in terms of ensuring complete removal of the bulb) in order to parallel the methods of the AC transection procedure. Because this procedure was less traumatic (e.g., less bleeding), memory tests could be performed after the transection, a necessity of the present experimental design.

\section{Training and Testing}

The pups received unilateral olfactory conditioning training before transections were made in order to establish a one-sided memory. Thirty minutes prior to training, a nose plug was placed in each pup, with the side being counterbalanced across litters. Just prior to training, the pups' bladders were emptied by gently stroking the anogenital region with a soft sable brush. The pups were then placed in a clear rectangular plastic container $(11 \times 6 \times 11 \mathrm{~cm})$ located in a warm test incubator $\left(32^{\circ} \mathrm{C}\right)$. The incubator was constructed from a 57 -liter glass aquarium covered with a roof of hinged Plexiglas panels (Hall, 1979). To deliver the cedar odor stimulus to a pup, a light stream of air was pumped (using an aquarium air pump) through plastic tubing into a closed 25 -ml flask containing $1 \mathrm{ml}$ of cedar wood oil (Fisher Scientific). A piece of tubing exiting from the flask extended into the pup's test container. Activating the pump immediately introduced an airstream bearing cedar odor into the pup's environment. A blower in the test container removed scented air.

During training, the pups received 10 trials consisting of a 20sec-long exposure to cedar odor, beginning $15 \mathrm{sec}$ before each infusion and continuing during the 5-sec intraoral infusion of sucrose $(15 \%)$ reinforcer delivered through the cannula. Cedar-milk pairings occurred every minute for $10 \mathrm{~min}$. Control pups received no training but had nose plugs inserted and switched in the same manner as conditioned pups. A single untrained control procedure was used in this experiment. In previous studies that included various conditioning controls, no differences in conditioned preference were found among backward, random, conditioned stimulus (odor)-only, unconditioned stimulus (oral infusion)-only, or naive control groups using this conditioning paradigm (Johanson \& Hall, 1982; Johanson, Hall, \& Polefrone, 1984). Unilateral conditioning has also been shown to be specific to the odor used during conditioning (Kucharski et al., 1986). Here, a single training condition and a single control condition were used because our interest was in exploring access routes to an established memory rather than features or properties of the learning process itself. One hour after training, the pups received transections or sham lesions.

Odor-preference memories were tested in the untrained side $4 \mathrm{~h}$ after the transection procedure. For testing, the pups were placed in a container that had fresh bedding (Ab-Sorb-Dri) on one side of the floor and fresh bedding scented with cedar odor on the other side (approximately $0.5 \mathrm{ml}$ of cedar wood oil was added to $85 \mathrm{~g}$ of bedding; the two sides of bedding were separated by a $1-\mathrm{cm}$ high plastic partition). Each preference test consisted of five 30 sec trials. For each trial, the pup was placed lengthwise on the partition dividing the two sides of the test container (direction of placement was alternated from trial to trial) and the cumulative amount of time the pup spent on the cedar-scented side of the container was recorded. A pup was scored as being over the cedar side if its snout was over the cedar-scented bedding (this test procedure is a slight modification of Johanson \& Teicher, 1980). Because our primary interest in this experiment was the presence of memory access from the untrained side, we tested this side first in all pups.

Following memory tests from the untrained side, nose plugs were switched and the pups were allowed $30 \mathrm{~min}$ to adapt to the new position. They were returned to the incubator for this adaptation. They were then tested for odor preference from the trained side. Note that, because of the potential absence of intact olfactory structures on the trained side in some groups, these later trials were of varying interest and significance.

\section{Histology}

Following the final test, the rats were sacrificed. Their brains were removed, frozen, sectioned horizontally in $20-\mu \mathrm{m}$ sections, and subsequently stained with thionin. The area of damage was reconstructed onto atlas figures (modified from Paxinos \& Watson, 1986), with particular attention paid to location of the transection in relation to the AC and olfactory-system structures. Reconstructions were made with regard to total extent of physical damage, necrosis, and gliosis. Working independently and blind to the pup's condition and performance, each experimenter assessed each lesion and rated its level and completeness.

\section{Additional Olfactory Peduncle Transections}

Because a number of the olfactory peduncle transections in the original series were classified as incomplete, an additional series of animals was prepared in which peduncle transections were placed slightly more posteriorly and in which the transection wire was swung twice to ensure a complete transection. Two to 3 pups were used from each of six litters, with at least 1 pup from each litter in the transection condition and 1 pup in the sham condition. Where there was more than 1 pup in a condition, these scores were combined to create a litter average for analysis. In this series, we also replicated our previous experiment (Kucharski \& Hall, 1987), which involved midline AC transections after training, in order to provide comparative data for the case in which all pups are tested first on the untrained side (1-2 additional pups from each of the above litters). The pups in this series were trained, tested, and lesioned as described above, with two exceptions: the peduncle transection was made with two complete passes of the needle; and the pups in this series were tested for responsiveness to a novel odor on the transected side after testing was complete. AC transections were made by inserting the transection needle $1 \mathrm{~mm}$ posterior to bregma, just lateral to the sagittal sinus, and to a depth of $7 \mathrm{~mm}$ perpendicular to the skull; the needle was then swung forward twice through 


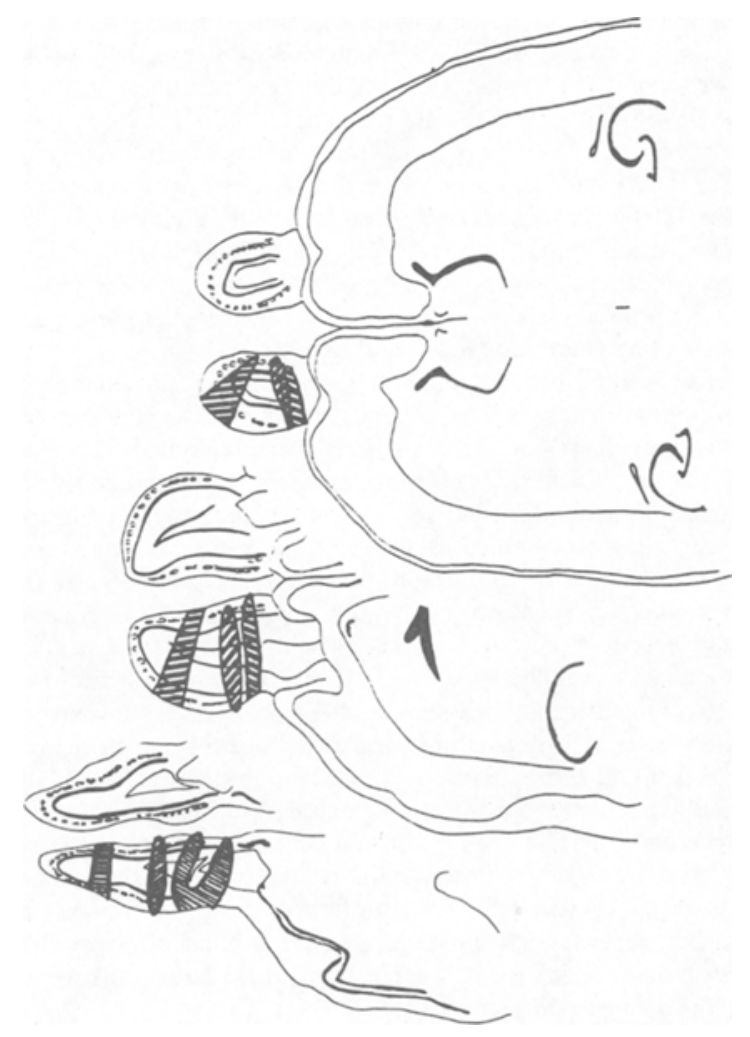

Figure 2. Diagram of representative complete olfactory peduncle transections in three horizontal planes. Cross-hatched outlines represent the extent of damage in individual animals. The most anterior transection is characteristic of the damage in 2 trained and 1 control pup; preference scores of these pups were similar to those of pups that had more posterior transections. A more typical level of transection is represented by the two more posterior examples.

an arc of $30^{\circ}$ in the sagittal plane. Left and right transections were alternated. After testing, the brains were processed for histology and the transections were evaluated as described above.

\section{Data Analysis}

Each training and transection group was represented by a single subject within each litter. Because of variations in the success of transections, however, a given litter did not necessarily have a full complement of pups with complete transections at each level. We treated litters as the experimental unit throughout these experiments (Denenberg, 1977, 1984), with treatment group (conditioned or naive) and transection level as factors for statistical analysis. Treatment effects on preference scores were evaluated by a separate ANOVA for scores for the untrained-side and the trained-side tests (procedure for unequal $n \mathrm{~s}$; Kirk, 1982). Comparisons between individual means were made by Tukey's HSD method (Kirk, 1982).

\section{RESULTS AND DISCUSSION}

\section{Location and Extent of Transections}

Figures 2,3, and 4 illustrate the nature and extent of the transections that were categorized as complete at each of the three levels (see Table 1 for the number of pups in each group). Olfactory peduncle transections categorized as complete had a continuous cut from the lateral to the medial margin of the bulb in each section examined (Figure 2). For 3 animals that had complete cuts, the transections were in the anterior portion of the bulb, as indicated in the figure. In addition, in some cases, our horizontally sectioned histological material was incomplete dorsally and/or ventrally; thus it is possible that a small portion of the lateral olfactory tract was left intact in some animals, leaving some output capability for the olfactory bulb on the trained side. However, with the exception of the pups with the most anterior cuts, it is likely that virtually all input to the bulb from the contralateral olfactory system, which ascends in the $\mathrm{AC}$, would have been eliminated.

Posterior limb transections (Figure 3) extended laterally from near the midline in the thalamus to the piriform cortex and frequently into the lateral olfactory tract. They produced extensive but variable diencephalic damage.

Anterior limb transections (Figure 4) generally produced damage to the nucleus accumbens and the anterior portion of the caudate putamen. There was little damage to any but the posterior division of the AON in these pups, and damage to the piriform cortex was more restricted than it was for the posterior limb transections. The level of this transection ranged from just rostral to the AON to a level just caudal to the division of the anterior and posterior limbs. We were able to further categorize pups into three subgroups reflecting the level of the transection within the anterior limb (approximate levels as indicated in Figure 4).

Transections categorized as incomplete in both commissure groups were similar to complete cuts in terms of tissue damage, but generally just missed the commissure or appeared to damage only a portion of it.

\section{Presence of Memories}

Memories were well established by the time of transection and were not nonspecifically disrupted by the transection procedures, as shown by clear evidence for memory in the sham-lesioned group and in several of the transected groups. Lack of disruption of memories by transections per se was also shown in earlier studies, in which AC transections performed 30 min to 5 days fol-

Table 1

Number of Subjects Categorized into Each Transection Group

\begin{tabular}{|c|c|c|c|c|}
\hline \multirow[b]{2}{*}{ Subjects } & \multicolumn{4}{|c|}{ Lesion } \\
\hline & Sham & $\begin{array}{l}\text { Olfactory } \\
\text { Peduncle }\end{array}$ & $\begin{array}{c}\text { Anterior } \\
\text { Limb }\end{array}$ & $\begin{array}{c}\text { Posterior } \\
\text { Limb }\end{array}$ \\
\hline \multicolumn{5}{|c|}{ Complete Transections } \\
\hline Trained & 16 & 11 & 13 & 11 \\
\hline Naive & 13 & 11 & 12 & 14 \\
\hline \multicolumn{5}{|c|}{ Incomplete Transections } \\
\hline Trained & & 4 & 4 & 2 \\
\hline Naive & & 6 & 1 & 2 \\
\hline
\end{tabular}

Note-One pup from each of 16 litters was initially designated for each of the experimental conditions. Because of incomplete transections, recategorization across transection group, and incomplete behavioral data, final sample size was not equivalent across groups. 
lowing training did not disrupt the storage or persistence of olfactory memories on the trained side (Kucharski \& Hall, 1988).

\section{Disruption of Memory Access from the Untrained Side}

Only transections of the anterior limb of the AC disrupted the retrieval of odor-preference memories from the untrained naris (Figure 5). When tested with their untrained naris open, trained pups with complete anterior limb transections spent less time over the cedar odor side of the test apparatus than did sham-lesioned pups that had been trained, and the trained pups showed the same low cedar preference as that shown by naive pups (training and transection effects, $F \mathrm{~s}>5.20$, $p \mathrm{~s}<.01$; trained anterior limb vs. sham, $p<.01$; no other post hoc comparisons were significant). In contrast, pups that received complete posterior limb transections and pups that received olfactory peduncle transections showed clear evidence of a conditioned odor preference from the untrained side, relative to their naive controls. These latter two tran- section groups were indistinguishable from sham-lesioned pups in their preference scores. Thus, an intact anterior limb appears to be essential to access contralateral olfactory preference memories, whereas neither posterior limb nor olfactory peduncle transections on the trained side disrupted the pups' ability to retrieve odor-preference memories when the stimulus was presented to the untrained side of the olfactory system.

The level of transection of the anterior limb did not appear to influence the disruptive effect of these lesions. Even though the number of pups categorized into each of the three anterior-posterior levels was relatively small ( $n s=6,4$, and 3 ), there was no indication in their preference scores of any potential difference between groups (scores $=50.3,55.5$, and $52.7 \mathrm{sec}$ ). Indeed, there was little variability in this anterior limb transection group in general, with all pups showing control-like, moderate aversion to the cedar odor.

It is worth noting that plugging a naris probably does not produce an absolute blockade of stimulation of the olfactory mucosa on the plugged side. Some flow or diffu-

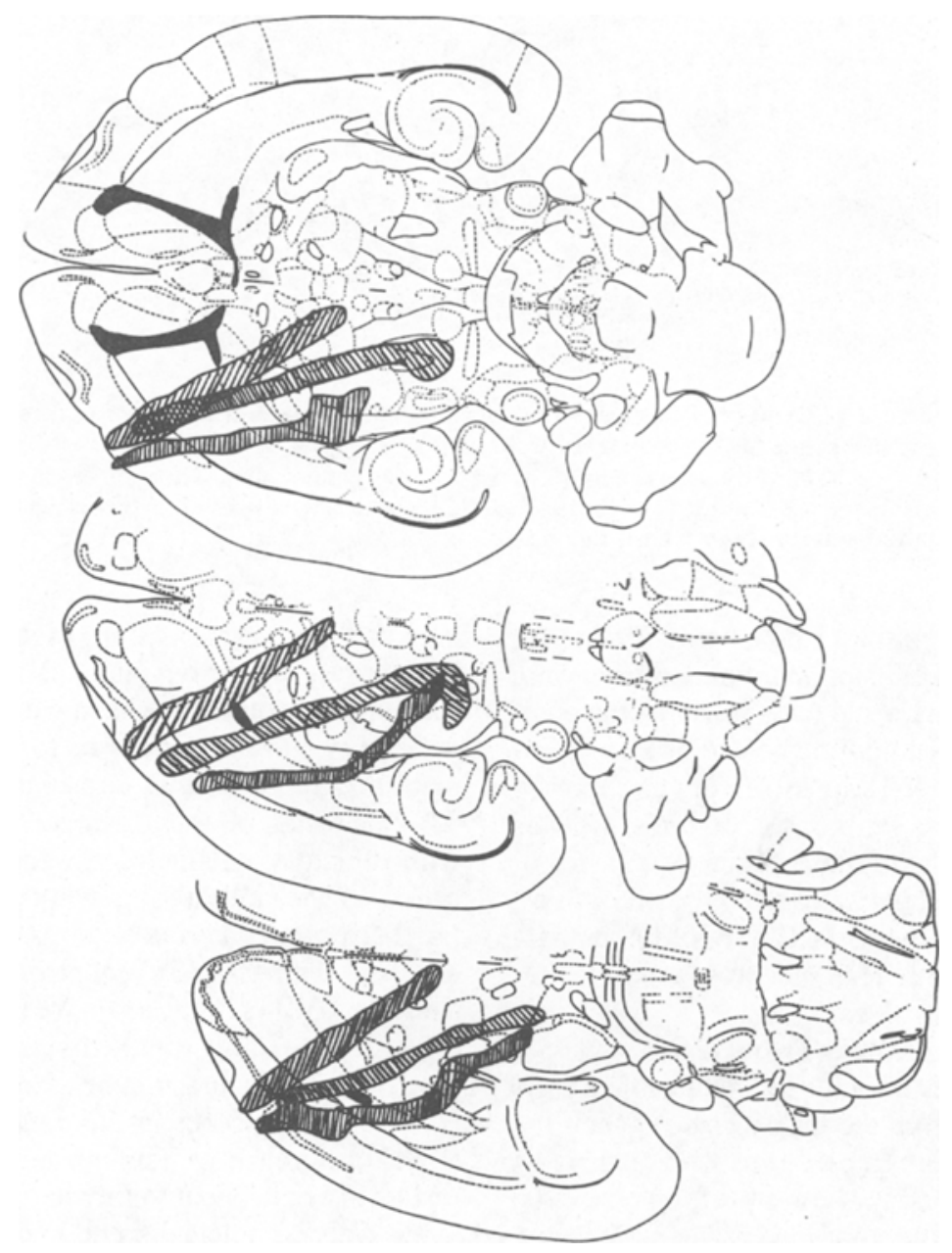

Figure 3. Diagram of representative complete posterior limb transections in three horizontal pianes (drawn on Figures 97, 95, and 93, modified from Paxinos \& Watson, 1986). Solid black regions are the limbs of the anterior commissure. 


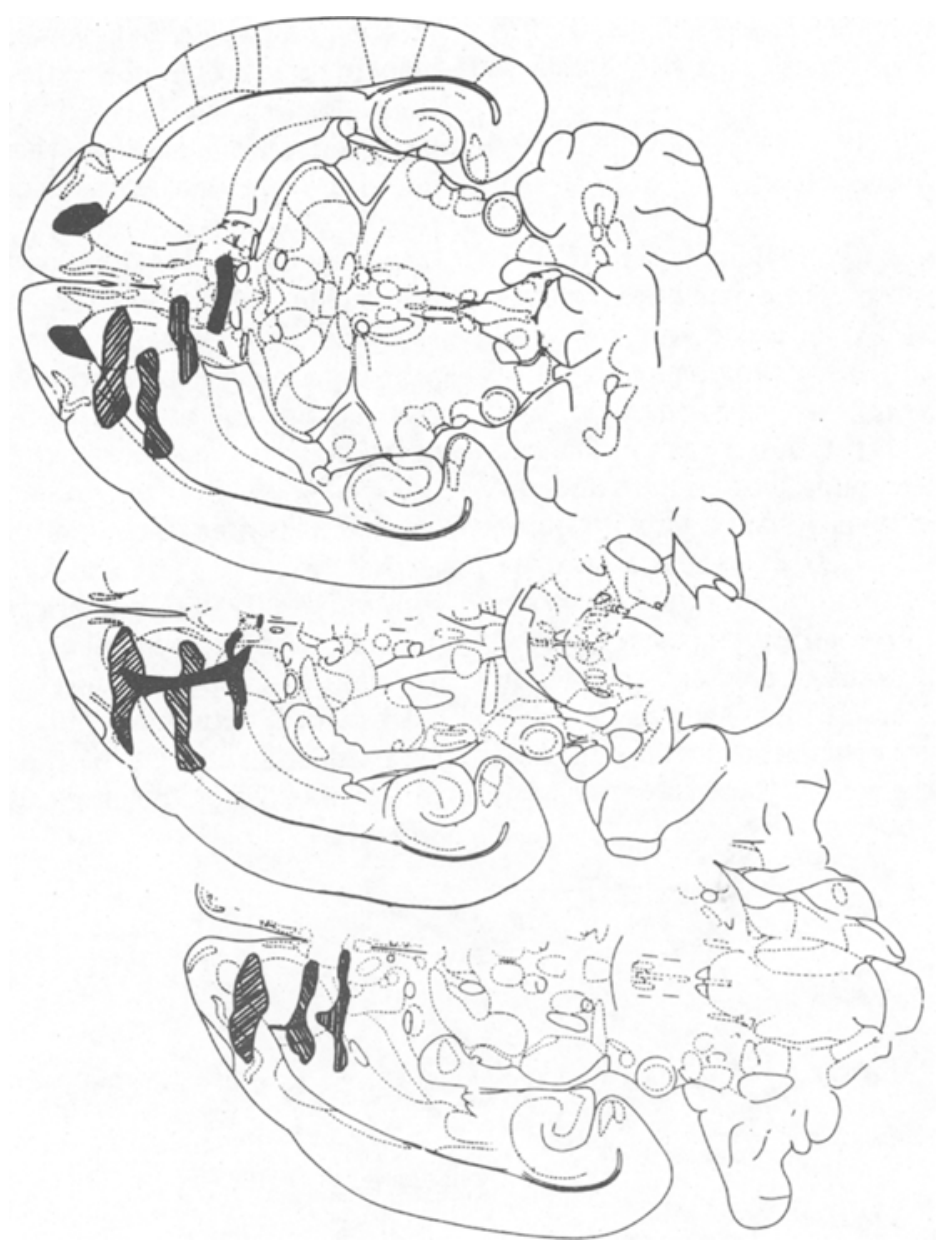

Figure 4. Diagram of representative complete anterior limb transections in three horizontal planes (drawn on Figures 99, 97, and 95, modified from Paxinos \& Watson, 1980). Solid black regions are limbs of the anterior commissure. These three transections are also illustrative of the three levels of cut that were used to further categorize the anterior limb transection data.

sion may occur between the sides of the nasal cavity. Yet the blockade was a functional one; no training effects were previously apparent in 12-day-old pups in which the commissure had been cut on the midline before or after training (e.g., see Kucharski \& Hall, 1987) or, as reported here, in which the anterior limb of the commissure was cut. We have also seen this same absence of transfer of odor-experience effects from one naris to the other in olfactory habituation experiments in 12-day-old pups with transected ACs (Hedrick \& Hall, in press).

\section{Effects on Responses from the Trained Side}

After the pups were tested with their untrained naris open, they were tested from the trained side. When the pups that had received sham transections were tested this second time, they continued to show a preference for the cedar odor $(78.8 \mathrm{sec}$ on the untrained side vs. $79.3 \mathrm{sec}$ on the trained side), as in our previous experiments. For pups with transections, interpretation of the effects must be cautious and provisional. Olfactory structures on the trained side would have been variably disrupted, and thus the ability to sense odors or to process olfactory information may have been damaged on this side.

When tested on their lesioned side, naive control pups with olfactory peduncle transections showed little indication of the normal slight initial aversion to the odor. Furthermore, preference scores for trained pups were no different than scores for control pups (trained vs. naive controls, 63.0 vs. $73.2 \mathrm{sec}$ ). We cannot be certain whether the failure to show any aversion or preference by these pups was due to anosmia or to an altered response to the odor, because scores in this range could reflect either a slight preference or random behavior. However, these results are consistent with what would be expected from a relatively complete disruption of olfaction on the trained side. Remember, though, that the olfactory preference 


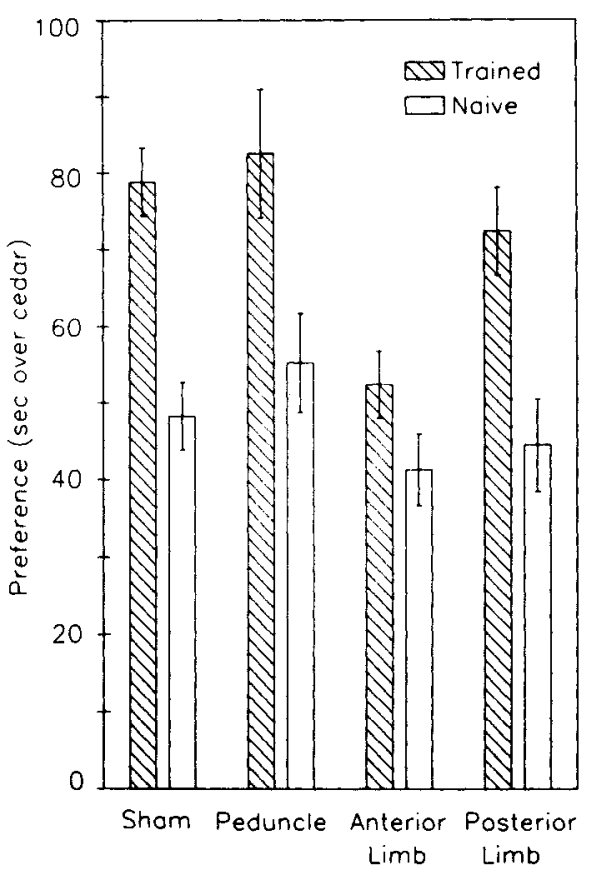

TRANSECTION LOCATION

Figure 5. Mean ( \pm SEM) preference scores (expressed as time spent over the cedar odor in seconds, out of $150 \mathrm{sec}$ ) for pups with transections categorized as complete at the level of the olfactory peduncle (see Level 1, Figure 1), in the anterior limb of the anterior commissure (AC; Levels 2-3), in the posterior limb of the AC (Level 4), and from control pups that received sham lesions. Open bars are scores for naive pups that did not receive preference training. Crosshatched bars are scores for pups in the experimental groups that were trained and received transections.

memory of the trained pups with peduncle cuts was not lost, as they showed good evidence of learning from the untrained side (e.g., see Figure 5).

The behavior of pups with posterior limb transections tested from the trained side was essentially identical to what they had shown on tests from the untrained side and was similar to that of animals that received sham lesions. Thus, these pups still seemed able to access the preference memory from both the untrained and the trained sides.

In pups with anterior limb lesions, the preference scores from testing on the trained side were variable and are potentially instructive. The scores for pups that had anterior limb transections and had been trained appeared to fall into three ranges (high, intermediate, and very low). Five of 13 of these pups showed a relatively strong preference (preference scores of $86-95 \mathrm{sec}$ ). Since only 1 naive animal had a preference score in this range, it seems reasonable to assume that some (or all) of these pups were still expressing the trained preference, even though none of these animals had shown a preference from the untrained side (scores of all 5 pups were lower on the untrained side than on the trained side). Thus, in these animals, the transection appeared to disrupt access to the memory, even though the memory was still present. Five other pups had intermediate scores when tested on the trained side $(41-74 \mathrm{sec})$. It is not possible to know what these scores represent individually, because they are in the range that could indicate a mild naive aversion, a slight learned preference, or simply random responding. This lack of distinction means that the memory itself may have been disrupted in these pups, and thus their deficits when tested on the untrained side could have been due to memory loss itself rather than to disruption of access to a still-present memory. Finally, 3 pups had very low preference scores $(21-28 \mathrm{sec})$. Because there was only one other score this low in the 29 sham-lesioned pups and 25 posterior-limb-lesioned pups, these anterior limb transections may have produced an exaggerated aversion to the cedar odor. Notably, 3 pups in the naive, anterior limb group also showed low preference $(15-33 \mathrm{sec})$ when tested on the lesioned side, as did the incompletely lesioned pup in this condition. Thus, the odor aversions seem to be a result of damage created by the lesion and not to be related to the pups' particular experiences. For this group, there appears to be no memory for the condi-

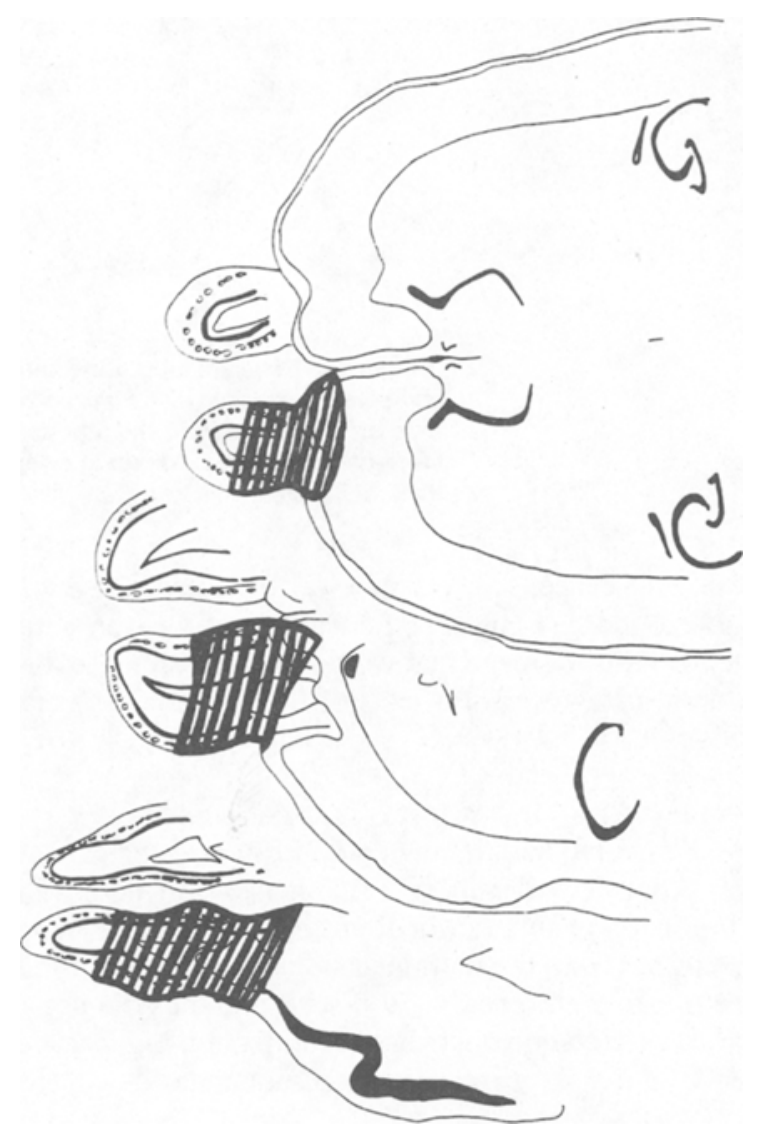

Figure 6. Diagram of the extent of complete olfactory peduncle transections for the second experiment in three horizontal planes. Cross-hatched outlines represent the total extent of damage in the 6 transected and trained pups. Damage in each individual animal was extensive. 


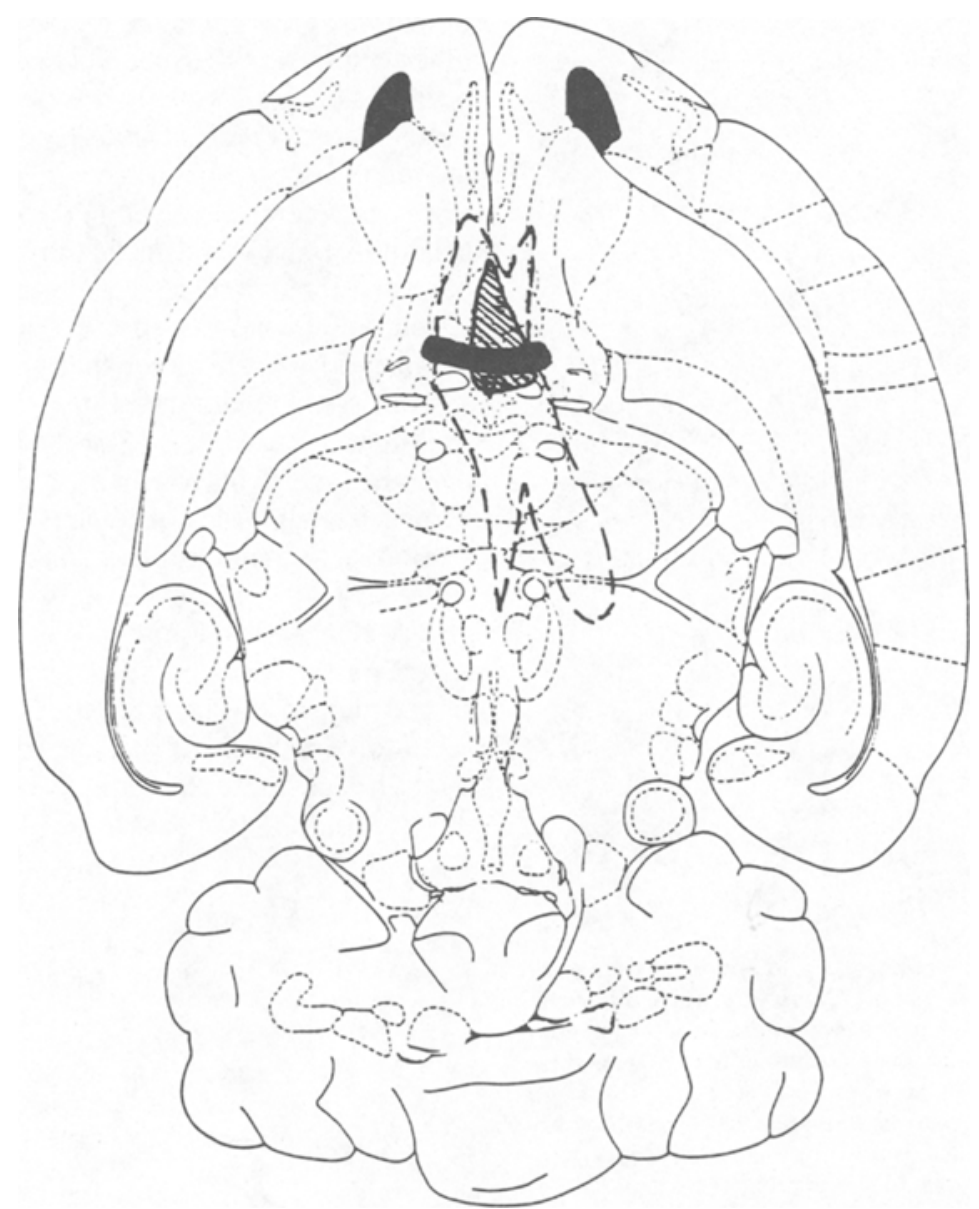

Figure 7. Diagram of midline anterior commissure $(\mathrm{AC})$ transections in the second experiment (drawn on Figure 99, modified from Paxinos \& Watson, 1980). Common area of damage to the 5 pups with complete transections is indicated by the cross-hatched region. Maximal extent of all damage is indicated by the dashed outline. $A C$ is solid black.

tioned preference, or at least no way to access it. We were unable to identify any specific features of the transection level or tissue damage that would distinguish these three different categories of effects of the anterior limb transections.

\section{Memory After Incomplete Transections}

A relatively small number of pups was categorized in each group as incomplete. Still, it is worth noting that in the incomplete anterior-limb-lesioned group, trained pups tested from the untrained side showed good preference (mean preference $=79.8 \mathrm{sec}$ ), and thus did not appear to suffer a memory disruption from the general effects of damage at this level transection.

\section{Additional Olfactory Peduncle Transection Series}

Because the number of complete olfactory peduncle transections from the first series was low and the level was somewhat variable, we prepared a second group of bulb transections as described above. In this series, we also included a replication of previous midline AC transection experiments to permit a direct comparison to our earlier results. The significant procedural difference in the olfactory peduncle transections was a double swinging of the transection needle to ensure thorough and complete cuts. Transection reconstructions for both groups of pups are provided in Figures 6 and 7.

The test results were consistent with those obtained from pups with complete olfactory peduncle cuts in the first experiment. When tested from the untrained side, trained pups still showed a relative preference for the conditioned cedar odor, and their behavior was indistinguishable from that of sham-lesioned controls [transection effect, $F(5,15)$ $=0.39$, n.s.; Figure 8]. In contrast, pups that received AC cuts showed the same slight aversion to cedar that was shown by naive animals (Figure 8 , inset). These findings thus complement those described above in showing that blocking access to the trained olfactory bulb did not prevent the pups from accessing previously stored olfac- 


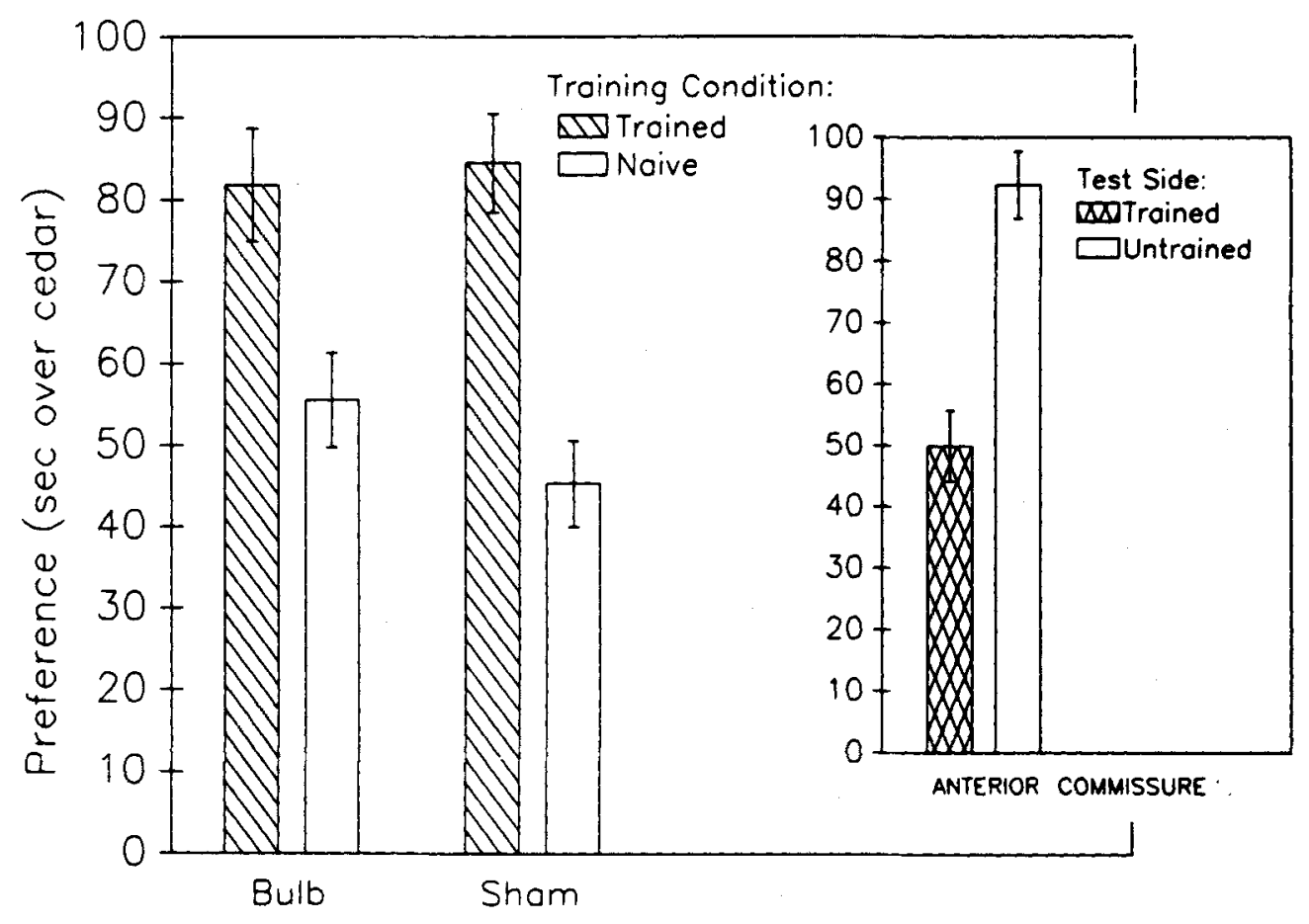

TRANSECTION LOCATION

Figure 8. Mean ( $\pm S E M$ ) preference scores for the second portion of the experiment, in which an additional group of pups (naive controls and trained pups) received olfactory peduncle transections and a comparison group received midline anterior commissure (AC) transections. For pups with the peduncle cut, these are preference scores from a test with the untrained naris open. For AC-transected pups (inset), these are preference scores from a first test with the untrained naris open, followed by a second test with the trained naris open. The latter comparison shows a lack of recall from the untrained side (e.g., compare to peduncle-transected pups; a replication of Kucharski \& Hall, 1987, 1988), but good preference memory from the trained side ( $\mathrm{s}$ = 5-6 per group).

tory preference memories. On the other hand, midline cuts to the AC prevented access to the conditioned odor memory from the untrained naris, as did the transections of the anterior limb described above and in previous reports.

\section{SUMMARY AND CONCLUSION}

The findings of this experiment, along with the preceding studies of unilateral odor-preference learning (Kucharski \& Hall, 1987, 1988; Kucharski et al., 1986), support a straightforward interpretation of localization of one form of olfactory memory. In young rats, odor-preference memory for stimuli restricted to one side of the olfactory system by occluding a naris is functionally stored on the stimulated side of the olfactory system. Unilateral storage of these training effects holds true even for 12-day-old pups, in which the memory can be accessed from the contralateral side. The results from the present experiments further indicate that it is the $\mathrm{AC}$ that provides the contralateral access, and that the anterior limb of the AC appears to provide the essential connection. Moreover, since even anterior cuts in the anterior limb (short of the level of the olfactory peduncle) eliminated access to the memory, it seems likely that projections to the $\mathrm{PCa}$ or the AON are the essential components.

This interpretation of a localized nature of olfactory memory and its lateralized storage is probably an oversimplification of the learning and storage process (cf. Freeman \& Skarda, 1985), yet it provides an initial parsimonious fit to the data. It is also possible to imagine a bilateral form of storage with asymmetrical retrieval components that might result in similar findings, but we suggest the first interpretation as a more heuristically useful starting point.

We further argue that the present results show that the disruption of memory access produced by AC transections close to the midline, as in previous studies and the second experiment here, is not a result of secondary damage to the corpus callosum or hippocampal commissure. In the present experiments, cuts in the anterior limb of the AC, which spared these structures, yielded essentially the same results.

More importantly, these findings appear to rule out the olfactory bulb as a necessary site for these types of olfactory preference memories in young pups. Even though 
some olfactory peduncle cuts may have been incomplete with respect to lateral olfactory-tract output from the bulb, it is likely that cuts classified as complete did transect the pathways to the bulb from the contralateral AON needed for memory retrieval via contralateral olfactory stimulation. This interpretation is consistent with other findings that show that an intact olfactory bulb need not be present for the retrieval of odor memories in young mammals (Hudson \& Distel, 1987; Lu \& Slotnick, 1989; Risser \& Slotnick, 1987a, 1987b). A site-specific form of functional and anatomical plasticity has been demonstrated in the olfactory bulb of developing rat pups (Coopersmith \& Leon, 1984; Woo, Coopersmith, \& Leon, 1987), but it occurs in the context of a different paradigm involving training over 18 days. In that paradigm, it is not yet known whether the identified changes in the bulb are essential to expression of the odor memory.

In short, we believe that we have identified a relatively specific projection that provides access to a form of early memory. How close the connections of the anterior limb are to the memories themselves is unclear; however, several recent theoretical statements suggest the potential for coding of such memories in the olfactory cortex (e.g., Granger, Ambros-Ingerson, \& Lynch, 1988; Haberly \& Bower, 1989). Thus, odor-preference memories may be localized to the AON or the anterior olfactory cortex, only a synapse or two from the olfactory bulb. How such memories are expressed in the output of odor-preference behavior remains to be explored.

\section{REFERENCES}

CoOPERSMith, R., \& LeON, M. (1984). Enhanced neural response to familiar olfactory cues. Science, 225, 849-851.

Denenberg, V. H. (1977). Assessing the effects of early experience. In R. D. Myers (Ed.), Methods in psychobiology (Vol. 3, pp. 127147). New York: Academic Press.

Denenberg, V. H. (1984). Some statistical and experimental considerations in the use of the analysis-of-variance procedure. American Journal of Physiology, 246, R403-R408.

Doty, R. W. (1973). Forebrain commissures and vision. In R. Young (Ed.), Handbook of sensory physiology (pp. 543-582). Berlin: Springer-Verlag.

FreEman, W. J., SKarda, C. A. (1985). Spatial EEG patterns, nonlinear dynamics and perception: The neo-Sherringtonian view. Brain Research Reviews, 10, 147-175.

Granger, R., Ambros-Ingerson, J., \& Lynch, G. (1988). Derivation of encoding characteristics of layer II of cerebral cortex. Journal of Cognitive Neuroscience, 1, 61-87.

HABERLY, L. B., \& BowER, J. M. (1989). Olfactory cortex: Model circuit for study of associative memory? Trends in Neuroscience, 12, 258-264.

Haberly, L. B., \& Price, J. L. (1978). Association and commissural fiber systems of the olfactory cortex of the rat. Journal of Comparative Neurology, 178, 711-740.

HALL, W. G. (1979). The ontogeny of feeding in rats: I. Ingestive and behavioral responses to oral infusions. Journal of Comparative \& Physiological Psychology, 93, 977-1000.

Hedrick, C., \& Hall, W. G. (in press). Developmental change in unilateral olfactory habituation is mediated by maturation of the anterior commissure. Behavioral Neuroscience.

Hudson, R., \& Distel, H. (1987). Regional autonomy in the peripheral processing of odor signals in newborn rabbits. Brain Research, 421, 85-94.

Johanson, I. B., HALL, W. G. (1982). Appetitive conditioning in neonatal rats: Conditioned orientation to a novel odor. Developmental Psychobiology, 15, 379-397.

Johanson, I. B., Hall, W. G., Polefrone, J. M. (1984). Appetitive conditioning in neonatal rats: Conditioned ingestive responding to stimuli paired with oral infusions of milk. Developmental Psychobiology, 17, 357-381.

JohanSON, I. B., \& TEICHER, M. H. (1980). Classical conditioning of an odor preference in 3-day-old rats. Behavioral \& Neural Biology, 29, 132-136.

KIRK, R. E. (1982). Experimental design: Procedures for the behavioral sciences. Belmont, CA: Brooks/Cole.

KuCharsKI, D., \& HALL, W. G. (1987). New routes to early memories. Science, 238, 786-788.

Kucharski, D., HaLL, W. G. (1988). Developmental change in access to olfactory memories. Behavioral Neuroscience, 102, 340-348.

KUCHARSKI, D., JohaNSON, I. B., \& HALL, W. G. (1986). Unilateral olfactory conditioning in 6-day-old rat pups. Behavioral \& Neural Biology, 46, 472-490.

LU, X.-C. M., \& SLotNick, B. M. (1989). Recognition and memory for propionic acid vapor in rats with lesions of olfactory bulb areas associated with 2-DG uptake. Association for Chemoreception Sciences Abstracts, 11, 182.

Luskin, M. B., \& Price, J. L. (1983a). The laminar distribution of intracortical fibers originating in the olfactory cortex of the rat. Joumal of Comparative Neurology, 216, 292-302.

LUSKIN, M. B., \& PrICE, J. L. (1983b). The topographic organization of association fibers of the olfactory system in the rat, including centrifugal fibers to the olfactory bulb. Journal of Comparative Neurology, 216, 264-291.

PAXINOS, G., \& WATSON, C. (1986). The rat brain in stereotaxic coordinates. Sydney: Academic Press.

Pedersen, P. E., Williams, C. L., \& Blass, E. M. (1982). Activation and odor conditioning of suckling behavior in 3-day-old albino rats. Joumal of Experimental Psychology: Animal Behavior Processes, 8, 329-341.

RiSSER, J. M., \& SLOTNICK, B. M. (1987a). Nipple attachment and survival in neonatal olfactory bulbectomized rats. Physiology \& Behavior, 40, 545-549.

RISSER, J. M., \& SLOTNICK, B. M. (1987b). Suckling behavior in rat pups with lesions which destroy the modified glomerular complex. Brain Research Bulletin, 19, 275-281.

SCHwob, J. E., \& Price, J. L. (1984). The development of axonal connections in the central olfactory system of rats. Journal of Comparative Neurology, 223, 177-202.

Suluvan, R. M., Hall, W. G. (1988). Reinforcers in infancy: Classical conditioning using stroking or intra-oral infusions of milk as UCS. Developmental Psychobiology, 21, 215-224.

Suluivan, R. M., Hofer, M. A., \& Brake, S. C. (1986). Olfactoryguided orientation in neonatal rats is enhanced by a conditioned change in behavioral state. Developmental Psychobiology, 19, 615-623.

Sullivan, R. M., \& LEON, M. (1987). One-trial olfactory learning enhances olfactory bulb responses to an appetitive conditioned odor in 7-day-old rats. Developmental Brain Research, 35, 307-311.

Woo, C. C., Coopersmith, R., \& LeON, M. (1987). Localized changes in olfactory bulb morphology associated with early olfactory learning. Journal of Comparative Neurology, 263, 113-125.

(Manuscript received September 17, 1989; revision accepted for publication February 2, 1990.) 